\title{
Private Cloud based Solution for the Implementation of a Virtual and Personal Learning Environment
}

\author{
Jimin Gao \\ Shen Zhen Polytechnic, Guang Dong Province, China \\ Gaojm@oa.szpt.net
}

Keywords: Online learning; Cloud Computing; Private Cloud; Google App Engine

\begin{abstract}
Online learning is a learning way to use online education platform for learners. It could let the learners start learning at anytime from anywhere by removing the traditional education's time and space limit. As the online learning style becomes more and more popular, its main base environment has gradually shown problems such as platform-independent platform's architecture, regional education information resources distribution model, information island issue between the learning platforms and other issues. The emerging of cloud computing makes it possible to solve these problems effectively.

Cloud computing is composed of a large number of different computer resources, through the distributed systems and virtualization technologies such as software and hardware virtualization, all resources could make a unified resource pool, then allocate computation, storage and other services to the customers by demand. At present, mature cloud computing platforms usually have over one million cluster servers, and can provide powerful computing capacity and huge amounts of storage space. This paper discusses building of a unified, open online learning system based on features of cloud computing service, and existing Google cloud computing platform - Google App Engine, aims to get rid of the defects of traditional online learning platform.
\end{abstract}

\section{Introduction}

Cloud Computing is a technology that can provide customers with highly scalable IT capacities through services via the Internet. It has brought new values to online learning activities, it provides a more reliable and efficient way. It has changed the way we develop and access applications. Applications (e.g. word processors, spreadsheets, hypermedia presentations, database systems) are delivered as internet services, where a scalable infrastructure can be utilized. These services can be accessed easily using a variety of web tools, while the applications and related data are hosted in the Cloud.

Clouds are built on top of many internet protocols, such as Web Services (WSDL, SOAP), and also some advanced Web 2.0 technologies such as REST, RSS, AJAX, etc.

In recent years, significant efforts have been made to introduce Cloud Computing based e-learning in educational institute. However, transfer from a traditional IT environment to a cloud environment is not a easy task. This paper presents a Private Cloud Computing-based solution for the implementation of a virtual and personal learning environment, using a wide range of cloud technologies.

\section{Some Basic Concept}

According to chronological order, the Cloud Computing is the 5th generation IT architecture in the IT world. These include: Mainframes architecture, Client-server architecture, Web architecture, SOA architecture and Cloud architecture. NIST( National Institute of Standards and Technology) have given an definition about the Cloud Computing architecture, and is considered an authoritative one. NIST pointed out that the cloud computing has three service models, four deployment models and five essential characteristics( as shown in Figure 1). 


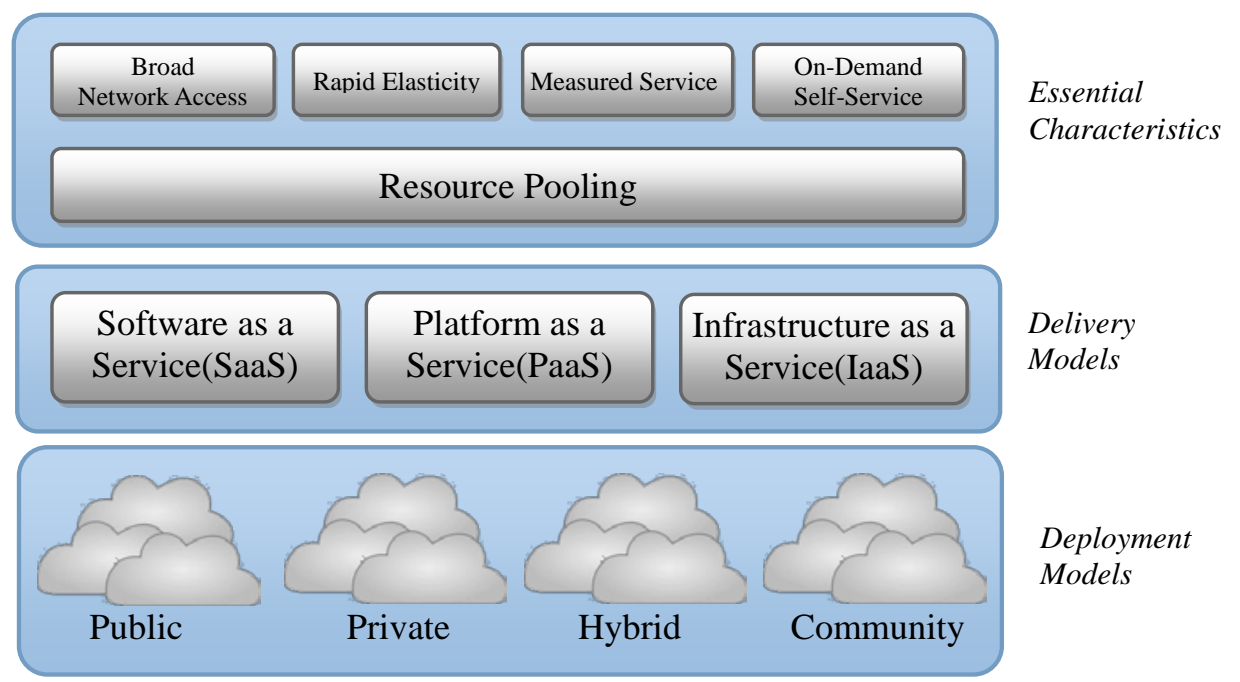

Figure 1. Visual Model of Cloud Computing Definition given by NIST

The characteristic of these service models:

- SaaS (Software as a Service): User of these service do not control or manage application platform as well as the underlining infrastructure. Cost of hardware and software maintenance can be greatly reduced.

- PaaS (Platform as a Service): User can develop and deploy his own applications on the provided platform, and management complexity is reduced. What development environment do you have depending on the PaaS provider.

- IaaS (Infrastructure as a Service): User can setup his own virtual machines on the platform and install his own operating system and application software. IaaS provide with the customers computing and storage resource services. thus customer avoids purchasing and managing the hardware and software infrastructure components.

Four deployments models involved:

- Public cloud: The cloud infrastructure is open to public users or some large groups and is owned by an cloud service providing organization.

- Private cloud: The cloud infrastructure is accessible only within the organization. It may be owned and managed by the organization itself.

- Community cloud: A kind of private cloud, that is shared by small customer groups with similar interests about data and applications.

- Hybrid cloud: It is a combination of public and private Cloud Computing model, using a public cloud for uncritical data or services, and using a private cloud for all the other sensitive data or services.

\section{E-learning System overview}

In this section, we are trying to give an outline of a E-learning system based on network architecture. Technologies of cloud computing are used to build a web interactive environment. In order to support both formal and informal learning, system is designed functionally and divided into three major functions. The first is the course management system, managed by the web server. The second is a variety of web services and applications using iGoogle as a application portal. We use a small agent to generation a personal learning path for learners. The third is a service engine we use to generate test for learners. To achieve personalization, two basic models are used, evaluation-based personalized learning and personalized learning space. We use a learning service to achieve collaborations, through this learning service learners are provided with special environment for collaborative purpose. Learners can turn on or off personalization on their own choice. This make it possible for learners to transfer their own personal learning space into sharing collaborative environment. One major task is to build relations between the learning content when 
adding new content to the knowledge base. In order to help learners to digest and understand specific topic, assessment questions and tests are automatic generated, because underlying architecture design support adding assessment questions for each topic, while at the same time allow building relationships between different learning content. We will make a detail discussion about the system in the following sections.

Managing Learning Content. Course content management component is designed to provide registered students who choose to learn specific course on line with a formal learning environment. The student has unlimited access to course materials that are maintained and uploaded by teachers. Material format about specific course varies according to the demand of the student. The material form can be a word documents, power point presentations which are hosted in Google Does, or lecture video recorded by the teacher. In Google Does, users can create their own documents, spreadsheets, and presentations. By default, these files are stored in Google server, and in prevailing document format (including OpenOffice, HTML, PDF, RTF, text files, Word) and can be downloaded and opened on the local computer. The creator of the document determines the document access privileges. The file you are editing will be automatically saved in order to prevent data loss, edit and update history will be recorded too. For organization and management purpose, the document can be classified and tagged. The Google document can be viewed, edit and Shared by multiple users at the same time. Video materials, which is easy to understand becomes more and more popular in teaching activities, also it can achieve better result compare to other format. Student can comment on specific videos, or even setup and join the relevant topic discussion groups to shared their experiences.

Personalized Learning Environment. There are many ways to establish a private learning environment within clouds. In the process of system development, we use gadgets as a platform to meet needs of the learners. Learners can build their private learning environment by them self to achieve their goals of study. For example, learners can access specific documents using Google Does gadgets, Messenger gadgets can also be used to send and receive instant messages, etc.

The most important gadgets above all is used to provide private network supporting environment. This gadgets is a collection and integration of learning resources, to make the learning process more exciting. The resources can be students, teachers, or group members who share common interest, or even electronic books, journals and files in media forms. Using this gadgets, we can bring experts, scholars in the field, all other teaching resources, and information together to build a private learning environment, and is technically supported By RSS Feeds. RSS (Really Simple Syndication) is a simple way for site to share information with other sites. RSS will collect and organize customized news, according time and format you want, sent directly to your computer. Learners will automatically receive customized information whenever new content added, thus make it possible for learners to track the latest knowledge.

Software Agent. The software agents is designed to setup learning conditions according to learner's knowledge background. There are two agents involved, the first is used to construct a individual learning path. It provides a dynamic, user-friendly interactive mechanism for the construction of suitable course to learners. Learners are requested to define and construct their own style of learning, as well as learning path using profiles. Learning materials are provided in the form of learning objects as network resources, which is defined by appropriate metadata stored in GBase. Metadata uses the IEEE standard LOM, it contains URI, topic, title, difficulty level, education role, concept and prerequisite concept. Using GBase API user can query metadata stored in the GBase with selection criteria and the specific topic as a condition to get a learning object lists.

The second agent is designed to generate quizzes with multiple choice questions. Google spreadsheets are used to store multiple choice questions. Each question contains some additional information, an unique identifier, related topic, related concept, and the difficulty level for the question. Using spreadsheet API application can generate automatically a set of questions for students. 


\section{Conclusion}

In this paper, we furthered the research and development on a Cloud Computing technology based virtual and personal online learning system. Cloud Computing provides a convenient method for us to build platform-independent applications. The mass scale storage provided by cloud, also makes it possible to the construction of the system. It provides a wide range of choice for formal as well as informal learning activities. The cloud computing at the same time, also makes the development of the application more easier, developers need to focus only on the development of the application itself, without having to consider issues about facilities and infrastructures.

The individual learning environment we proposed, involves a large number of Cloud Computing services, making the user interface more friendly and convenient to use. Contents of the course can be monitored in the learning process. Students can communicate and exchange ideas about related topics of the specific subject on the platform. Service oriented structure ensures the management of the learning resources, and the scalable network resources also can be utilized. The structure made a good combination of teaching and learning process. User can get more exciting experiences in the environment compared to the traditional teaching methods and processes. the flexibility and diversity of the use of teaching resources also have obvious advantages over the old ones. The tools we used in the learning process to access learning resources are familiar to every one. They can access learning materials with a variety of terminal device, including mobile device. In the learning processes, after course practices and self-tests can be conducted online too. All this makes it more convenient to reach one's different learning goals. We believe that it will have greater development and utilization values through further improvement.

\section{References}

[1] Leitner, L., \& Cane, J. (2005). A virtual laboratory environment for online it education. SIGITE '05. 283-289.

[2] Li, C., Toderick, L., Li, P., Mohammed, T., \& Lunsford, P. (2008). Networking lab simulation using virtual technologies. American Society for Engineering Education.

[3] Lunt, B., Ekstrom, J., Gorka, S., Hislop, G., Kamali, R., Lawson, E., LeBlanc,R., \& Reichgelt, H. (2008). Curriculum guidelines for undergraduate degree programs in information technology. Association for Computing Machinery IEEE Computer Society.

[4] Pickard, J., Spence, J., \& Lunsford, P. (2012). IPv6 certification and course development. SIGITE '12. 156-160.

[5] Doelitzscher, F., Sulistio, A., Reich, C., Kuijs, H., \& Wolf, D. (2010). Private cloud for collaboration and e-Learning services: from IaaS to SaaS. Computing,91(1), 23-42. doi:10.1007/s00607-010-0106-z.

[6]Brown, S. (2008) 'From VLEs to learning webs: the implications of Web 2.0 for learning and teaching', Interactive Learning Environments.

[7]Chakchai, S-I., Jain, R., Subharthi, P. and Pan, J. (2010) 'Virtual ID: ID/locator split in a mobile IP environment for mobility,multihoming and location privacy for the next generation wireless networks', International Journal of Internet Protocol Technology, Vol. 5, No. 3, pp.142-153. 